\title{
An Einstein manuscript on the EPR paradox for spin observables
}

\author{
Tilman Sauer \\ Einstein Papers Project \\ California Institute of Technology 20-7 \\ Pasadena, CA 91125, USA \\ tilman@einstein.caltech.edu
}

March 6, 2007

\begin{abstract}
A formulation by Einstein of the Einstein-Podolsky-Rosen incompleteness argument found in his scientific manuscripts is presented and briefly commented on. It is the only known version in which Einstein discussed the argument for spin observables. The manuscript dates, in all probability, from late 1954 or early 1955 and hence also represents Einstein's latest version of the incompleteness argument and one of his last statements on quantum theory in general. A puzzling formulation raises the question of Einstein's interpretation of space quantization and the non-classical spin degree of freedom.
\end{abstract}

\section{Introduction}

It is well-known that the EPR paper (Einstein, Podolsky, and Rosen, 1935) was actually written by Boris Podolsky "after many discussions," and that Einstein, as he confided in correspondence with Erwin Schrödinger, was discontent with the way it came out since he thought that "the main point was, so to speak, buried by erudition." ${ }^{1}$ Einstein gave his own version of the

\footnotetext{
${ }^{1}$ Einstein to Schrödinger, 19 June 1935, Albert Einstein Archives (AEA) call number 22-047, cited in (Howard, 1985, p. 175), see also the discussion by Fine (1996, p. 35) who
} 
EPR incompleteness argument in the same letter to Schrödinger and shortly thereafter in print in his essay on "Physics and Reality," (1936) where it is explicitly referred to as a "paradox" (p. 376). ${ }^{2}$

There are three more published versions of the EPR incompleteness argument by Einstein. One was included in his "Autobiographical Notes," drafted in 1947 and published in Schilpp's Albert Einstein Philosopher-Scientist in 1949, another in his "Reply to Criticisms" in the same volume, the third in his paper on "Quantum-Mechanics and Reality" which appeared in 1948 in a special issue of the journal Dialectica (Einstein, 1949a, pp. 83-87; 1949b, pp. $681-682 ; 1948)$. Other versions of the argument are found in his correspondence. $^{3}$

All of Einstein's own formulations of the EPR argument as known discuss the problem either directly for position and momentum observables, or else in terms of some set of non-specified canonically conjugate observables. In these known formulations Einstein illustrated the incompleteness argument by referring to a hypothetical experiment in which the values of the position and momentum variables of two spatially separated but quantummechanically entangled particles are being measured resp. inferred. Modern discussions of the EPR argument, however, routinely reformulate the EPR experiment for particle observables of a finite, usually 2-dimensional, Hilbert space and illustrate the experiment in terms of spin observables or for the polarization degrees of freedoms of photons. It is also for correlated photons that the EPR thought experiment was first realized in actual experiments. ${ }^{4}$

The first published reformulation of the EPR argument for spin observables seems to have been given in 1951 by David Bohm in his textbook on quantum mechanics (1951, pp. 614-622). ${ }^{5}$ Bohm considered his reformu-

was the first to point out the significance of this letter.

${ }^{2}$ Note that, as pointed out by Fine (1996, p. 47, n. 11), it is explicitly but erroneously denied by Jammer (1974, p. 186) that the authors of the EPR paper ever considered their argument as "paradoxical."

${ }^{3}$ See, e.g., Einstein to Schrödinger, 19 June 1935 (cp. note 1) and Einstein to J.L.B. Cooper, 31 October 1949 and 18 December 1949 (AEA 8-412, 8-414, cited in part by Stachel $(2002$, p. 391, 410f)). Discussions of Einstein's incompleteness argument with extensive references to his correspondence are given by Fine (1996, ch. 4), Howard (1985), (1990), and also Stachel (2002, ch. VI).

${ }^{4}$ Shimony (2006) provides a recent review of both the theory and the experiments performed.

${ }^{5}$ See (Jammer, 1974, p. 235). It is clear from the extant Einstein-Bohm correspondence that Einstein knew of Bohm's book but it is unclear to what extent if at all he had actually 
lation "conceptually equivalent" to the EPR version but argued that it was "considerably easier to treat mathematically" (p. 614). Bohm's reformulation also was presented just shortly after a mathematical difficulty of the original EPR argument had been pointed out by the mathematician J.L.B. Cooper (1950). ${ }^{6}$ Cooper had argued that spatial separation of two particles would imply an infinitely high potential barrier between the two spatially separated particles, leading to a vanishing of the joint wave-function at some place, say $z=0$. Such vanishing would render the momentum operator no longer selfadjoint (but still Hermitian), since its domain would have to be restricted to the positive (resp. negative) half line $[0, \infty]$ (resp. $[-\infty, 0]$ ). Therefore, Cooper argued, EPR were not allowed to invoke the respresentation theorem for the restricted momentum operator, which would have no (non-trivial) eigenfunctions at all. Einstein's response to this argument, given in correspondence after having read a manuscript version of Cooper's paper, was to repeat the incompleteness argument, emphasizing that the spatial separation of the two particles only demanded the vanishing of the wave function at $z=0$ in some limiting sense, and that hence the question of an infinite potential barrier was "of no interest" for the question. ${ }^{7}$

We now present and briefly comment on a manuscript in which Einstein gives another formulation of the EPR incompleteness argument. This appears to be the only version in which he discusses the EPR argument explicitly for spin observables.

\section{The manuscript}

Einstein's version of the EPR incompleteness argument for spin observables is found on the lower half of the verso of a sheet of calculations (AEA 62$575 \mathrm{r}$ ) that is part of a considerable batch of some 1800 pages of manuscript calculations. The manuscript pages turned up when Einstein's papers were being packed up to be shipped from Princeton to Jerusalem in $1982 .{ }^{8}$ Most of

read it, see the discussion below.

${ }^{6}$ See (Jammer, 1974, pp. 236-238). Jammer also discusses other early contentions of the EPR argument that amount to the charge of a wrong or inconsistent application of the mathematical formalism, such as Paul Epstein's objection that the argument does not take into account the time-dependence of the wave functions explicitly.

${ }^{7}$ See note 3 .

${ }^{8}$ The pages were then added to the Albert Einstein Archives at the Hebrew University of Jerusalem and given archival numbers 62-001 through 63-416 (Sauer, 2004). 
the manuscript pages show calculations in the context of Einstein's search for a unified field theory or else on problems of conventional general relativity. A few pages deal with other problems, but I found no other instance in the set that appears to be related to the quantum incompleteness argument. The whole batch contains manuscripts dating from the late twenties until the very end of Einstein's life. The dating of the particular sheet to be discussed here is unclear. The handwriting suggests a date late in Einstein's life as does the context of the unified field theory calculations. ${ }^{9}$ The most concrete hint is the fact that the particular sheet is found right after a sheet that can be dated explicitly as after 30 November 1954. This sheet (AEA 62-574) is a letter of Serge Moguillanes to Einstein, of that date, with calculations by Einstein on the back. ${ }^{10}$ It is unclear whether the two sheets are related in any other way that would entail a temporal proximity but a preliminary global assessment of the full batch of manuscript calculations has shown that, in general, proximity in the physical sequence of the sheets also reflects temporal proximity (Sauer, 2004, pp. 161-163). If we accept the date suggested by the proximity to the dated letter, late 1954 or early 1955, the manuscript would, most likely, represent Einstein's latest version of the incompleteness argument and one of his last statements on quantum mechanics in general. In any case, it is likely that the manuscript was written after Einstein's last published discussion of quantum mechanics (1953) and a fortiori later than Bohm's reformulation of the EPR argument in 1951.

The following is an English translation of the relevant passage of the manuscript: ${ }^{11}$

\footnotetext{
${ }^{9}$ The surrounding calculations on the recto and verso of the same sheet are dealing with the problems of finding a diagonal representation of the metric tensor and the positivity of the respective metric components, and with variational calculations involving the curvature tensor. Without further context, one might perhaps argue that these calculations may well have been done in the framework of conventional general relativity. However, notational continuities with the surrounding pages suggest that they were rather part of considerations in the context of Einstein's unified field theory approach based on an asymmetric metric.

${ }^{10}$ The letter (AEA 62-574-1) was typewritten in French and sent from Paris. With this letter, Moguillanes sent Einstein a copy of a book he had written, entitled "Néodémocratie," and asked for comments. I have not seen a copy of this book. The library catalogue of the Bibliothéque Nationale lists one book by S. Moguillanes that carries this title, but the bibliographic reference in the library catalogue lists the item as being self-published without a date. I have no other information about the author or his book.

${ }^{11}$ For ease of understanding, the following English translation incorporates words and
} 
Composite system of total spin 0.

1) The description is assumed to be complete.

2) A coupling of distant things is excluded.

If the spin of the subsystem $\mathrm{I}$ is measured along the $x$-axis, it is found to be either 1 or -1 in that direction. It then follows that the spin of the subsystem II equals 0 along the $y$-direction. But if instead the spin of subsystem I is measured along the $y$-direction, it follows that the spin of the subsystem II is equal to 1 or -1 .

If there is no coupling, then the result of a measurement of the spin of subsystem II may in no way depend on whether a measurement was taken of subsystem I (or on what kind of measurement).

The two assumptions therefore cannot be combined.

If the description is not assumed to be complete for the individual system, then that what is being described is not a single system but an ensemble of systems. Then a measurement of subsystem I amounts to the selection of a subensemble of the ensemble of the total system. Then the prediction for a measurement of subsystem II can depend on the choice of the measurement of subsystem I.

The conclusion is valid under the assumption that the assertion of quantum theory is correct, which we can hardly put into doubt.

The following lines were written at the right margin of the page:

a) the description by the quantum theory is an incomplete one with respect to the individual system, or

b) there is an immediate coupling of states of spatially separated things.

\section{Discussion}

Einstein's known versions of the EPR incompleteness argument have been discussed at length in the literature. ${ }^{12}$ To the extent that the argument is,

characters that I have added to the original text in order to render the words unambiguous and the sentences grammatically complete. The full German text is given in the appendix where all added characters and words are indicated by square brackets.

${ }^{12}$ See note 3 and further literature cited in these references. 
in fact, quite similar to other versions, it is not the purpose of this note to add to the existing historical commentary.

A puzzling feature of the argument is Einstein's assertion that the $y$ component of the spin of particle II would be "equal to $0 "$ " " $=0$ ") if the $x$-component of the spin of particle I is being measured. The assertion is puzzling since it is made for an individual system rather than for an ensemble of systems. If the two subsystems are in an entangled state with total spin 0 and the $x$-component $s_{x}^{\mathrm{I}}$ of the spin is measured on subsystem I, subsystem II will be in an eigenstate of $s_{x}^{\mathrm{II}}$ and not in an eigenstate of $s_{y}^{\mathrm{II}}$. Hence, a measurement of the $y$-component of the spin of subsystem II cannot give $s_{y}^{\mathrm{II}}=0$, which can emerge only as an average over an ensemble. Even though Einstein explictly speaks about the "result of a measurement of the spin of subsystem II" in the following sentence, he presumably has in mind not what measurements would yield, but what is the "real" state of affairs. If the total spin of system I is in the $x$-direction, the total spin of system II is also in the $x$-direction; which implies that it "really" cannot have a component in the $y$ direction. The passage thus raises the question of Einstein's understanding of space quantization and the non-classical spin degree of freedom. Somewhat suprisingly, we have very little textual evidence that would shed light on this question. Without pretending to provide an answer, I will briefly comment on relevant evidence that I am aware of.

The classical experiment demonstrating space quantization is the SternGerlach experiment (Gerlach and Stern, 1922). ${ }^{13}$ In this experiment silver atoms are deflected in two directions upon traversing an inhomogeneous magnetic field. The results showed that the spatial orientation of the magnetic moments of silver atoms in a magnetic field was quantized. Numerically, the quantization was later explained in terms of an electron spin that gives rise to the magnetic moment of the silver atoms. Immediately after Gerlach and Stern published their results, Einstein and Paul Ehrenfest (1922) critically discussed some implications of the experiment and some possible ways of accounting for the result without, however, providing themselves a positive explanation. Einstein and Ehrenfest understood that the magnetic moments of all silver atoms were aligned along the axis of the magnetic field and that

\footnotetext{
${ }^{13}$ The sequence of authors on the relevant papers is alphabetical. It is unclear to me why the experiment is usually referred to as "Stern-Gerlach," as is done, e.g., already in the title of (Einstein and Ehrenfest, 1922). One possible reason is that the experiment realized an idea originally published by Stern alone. To avoid confusion, I will continue to refer to the experiment in the usual parlance.
} 
they would do so without any mutual interaction (p. 31).

Several years later, in a letter to Ehrenfest dated 21 January 1928, (AEA 10 173) i.e. after the development of mature quantum mechanics and the recognition of the existence of the electron's spin, Einstein comments on the Stern-Gerlach experiment again. In the letter, he reported that he had suggested to Stern to do two new experiments. In the first one, which he calls "curious" ("lustig"), two Stern-Gerlach magnets would be put next to each other in such a way that the magnetized molecules would run first through one inhomogenous magnetic field, then through a stretch of empty space, and then through a second Stern-Gerlach device. The point of the experiment is that Einstein suggested that one would see no effect if the direction of the second magnetic field were reversed with respect to the first. ${ }^{14}$ The reasoning here is as follows. "In a magnetic field, let the molecular axis be aligned and follow the magnetic field if it changes slowly." 15 Apparently Einstein assumed that the molecule would follow the reversal of the two magnetic fields between the two Stern-Gerlach devices adiabatically: "If a molecule traverses two inhomogeneous fields that point in opposite directions, it will reverse itself on travelling through the interval between the two fields." 16

In a second experiment, Einstein suggested to Stern that he should try to separate the effects of a magnetic field gradient and of the magnetic field itself. "The paradoxical result to be expected is that, for a given field gradient, an arbitrarily small field should determine Stern's deflections (the plane of splitting)." 17

In late 1933 and early 1934, Einstein published several papers together with Walther Mayer on the use of the so-called semi-vectors for an alternate equivalent representation of the Dirac equation (van Dongen, 2004). These semi-vectors are a technique for representing spinors. The episode therefore at least indicates that Einstein was aware of contemporary mathematical techniques to represent spinors. Other than that the episode seems to shed

\footnotetext{
${ }^{14}$ The experiment is strongly reminiscent of Rabi's molecular beam apparatus, except for the lack of focussing diaphragms and, more importantly, of an oscillatory magnetic field that would induce the spin flips.

15 "Im Magnetfeld soll die Molekül-Axe orientiert sein und dem Magnetfeld bei dessen langsamer Veränderung nachfolgen."

16 "Durchläuft ein Molekül zwei entgegengesetzte inhomogene Felder, so kehrt es sich beim Durchlaufen des Intervalls zwischen beiden Feldern um."

17 "Das paradoxe zu erwartende Resultat ist, dass bei gegebenem Feldgradienten ein beliebig schwaches Feld für die Stern'schen Ablenkungen massgebend sein soll (Aufspaltungsebene)." (Einstein's emphasis)
} 
little light on our specific question.

Let me finally add a few comments concerning David Bohm's work and Einstein's reaction to it. The extant correspondence between Einstein and Bohm dates from ca. 1951 to November 1954. I have found no direct indication in this correspondence that Bohm's reformulation of the incompleteness argument for spin observables was explicitly discussed. Einstein certainly knew of Bohm's "excellent book about quantum theory"18 (Bohm, 1951), and Bohm's "causal" interpretation of quantum mechanics that he developed soon after publishing this book is discussed to some detail. ${ }^{19}$ There is, however, an indirect hint to the problem which also corroborates our dating of Einstein's manuscript. On June 8, 1954, Bohm sent Einstein the manuscript of a paper, co-authored with Jean-Pierre Vigier, which he had just submitted for publication. ${ }^{20}$ In the accompanying letter, Bohm also announced "some interesting new results" which he promised to communicate to Einstein as soon as they would be ready. These new results, he added in brackets, concerned "the theory of the spinning electron" (Bohm to Einstein, June 8, 1954, AEA 8-045). There are no indications in the further correspondence about these new results but the paper itself has two footnotes that are interesting in our context. In the paper, the authors discuss and extend a hydrodynamic model in which the Schrödinger equation is interpreted in terms of a continuous fluid and "the quantum potential may be thought of as arising in the effects of an internal stress in the fluid" (Bohm and Vigier, 1954, p. 209). A little later in the paper, the authors discuss the possibility that the velocity field may not be derivable from a potential and then add a footnote, in which they observe that "vortex components of the velocity may also explain the appearance of 'spin'," which, however, would be neglected at the present level of precision.

In the concluding paragraph of this paper, Bohm and Vigier discuss the

\footnotetext{
${ }^{18}$ Einstein to Nathan Rosen, March 11, 1954, AEA 8-042.

${ }^{19} \mathrm{An}$ appreciable fraction of the Einstein-Bohm correspondence (AEA 8-001 to 8-058) deals with Einstein's support for Bohm when the latter had left the United States, after refusing to "answer official questions concerning colleagues" (AEA 8-003). A number of items of correspondence in the cited archival call number range are letters of recommendation for Bohm.

${ }^{20}$ (Bohm and Vigier, 1954) was received by Physical Review on June 14, 1954. The typescript version sent to Einstein carries the archival number AEA 8-046. I did not see any marginalia in (a copy of) the manuscript, and the two footnotes to be discussed below (as well as the text in general) is the same in both the manuscript and the published paper.
} 
difference between the "usual" and the "causal" interpretation of quantum mechanics with respect to "the irregular statistical fluctuations in the observed results [...] when we make very precise measurements on individual atomic systems." According to the usual interpretation, these fluctuations are assumed to be "fundamental elements of reality" and "cannot be traced to anything else." At this point, we find the following footnote:

For example, they cannot in general be ascribed to the uncontrollable actions of the measuring apparatus, as demonstrated by Einstein, Rosen, and Podolsky, Phys. Rev. 47, 774 (1933) [sic!] and also D. Bohm, Quantum Theory (Prentice Hall Publications, New York, 1951), p. 614. As Bohr has made clear [Phys. Rev. 48, 696 (1935)] the measuring apparatus plus observed object must be regarded as a single indivisible system which yields a statistical aggregate of irregularly fluctuating observable phenomena. It would be incorrect, however, to suppose that these fluctuations originate in anything at all. They must simply be accepted as fundamental and not further analyzable elements of reality, which do not come from anything else but just exist in themselves. For a complete discussion of this problem, see, $\mathrm{Al}$ bert Einstein, Philosopher-Scientist, Paul Arthur Schilpp, Editor (Library of Living Philosophers, Evanston, 1949). (p. 215)

Einstein who was asked by Bohm for comments on this paper must surely have pondered over this footnote which puts together the relevant references, including a reference of the original EPR paper right next to the relevant page in Bohm's book. The existing later correspondence between Einstein and Bohm - two letters by Bohm, dated October 18 and November 14, 1954, and two letters by Einstein, dated October 28 and November 24, 1954, respectively - indeed discusses differences in opinion regarding the foundations of quantum mechanics. But these differences are expressed on a very general level, notably with respect to the question of whether one should give up the notion of a continuum, and the letters do not make any explicit reference to the EPR incompleteness argument or the problem of the spin degree of freedom.

A closer comparison of Einstein version of the incompleteness argument presented here and the discussion in (Bohm, 1951) should be embedded in a more detailed and comprehensive discussion of both Einstein's and Bohm's perspectives on quantum mechanics. The purpose of this note was to present 
an important document that adds to our understanding of these issues. It remains a deplorable fact, that Einstein himself would never explain his understanding of the quantum incompleteness argument in more explicit mathematical terms. But the succinctness of Einstein's incompleteness argument in the manuscript discussed here may also carry a positive aspect. It provides another concise statement of what, in Einstein's understanding, the EPR argument is about.

\section{Appendix: Partial transcription of AEA 62- $575 \mathrm{r}$}

Zus[ammengesetztes] System von Ges[amt] Spin 0.

1) [Die] Beschr[eibung ist als] vollst[ändig] vorausges[etzt].

2) [Eine] Koppelung distanter Din[g]e [wird] ausgeschl[ossen].

Wenn an [dem] Teilsystem I [eine] Mess[ung] des Spin[s] in [der] $X$ Axe vorgenommen [wird], dann ist [der] Spin dieses Teilsystems entweder 1 oder -1 in dieser Richtung[.] Dann folgt, das[s der] Spin des II-Teilsystem[s] $=0$ in der $Y$-Richtung [ist]. Wenn aber statt dessen an [dem Teilsystem] I [der] Spin in der $Y$-Richtung gemessen wird, so folgt, dass hierauf [der] Spin des II-Teilsystems [in der $Y$-Richtung] -1 oder +1 ist.

Wenn [es] keine Kopplung [gibt], so darf das Ergebnis ${ }^{21}$ einer Messung des Spins an [dem Teilsystem] II überhaupt nicht davon abhängen[,] ob überhaupt eine Messung an [dem Teilsystem] I vorgenommen war (bezw. was für eine Messung).

Beide Annahmen lassen sich also nicht vereinigen.

Wenn [die] Beschr[eibung] nicht als vollst[ändig] für das individuelle System $^{22}$ vorausgesetzt [wird], dann ist das Beschriebene nicht ein System, sondern eine System-Gesamtheit. Dann bedeutet [eine] Messung an [dem Teilsystem] I die Aussonderung einer Teilgesamtheit für das Ensemble das Gesamtsystem. $^{23}$ Dann kann die Voraussage für [eine] Messung an [dem Teilsystem] II von der Wahl der Messung an [dem Teilsystem] I abhängen.

\footnotetext{
${ }^{21}$ The words "das Ergebnis" are interlineated.

${ }^{22}$ The words "als" and "für das individuelle System" are interlineated.

${ }^{23}$ The words "das Ensemble" are interlineated, and "das Gesamtsystem" should probably read "des Gesamtsystems".
} 
[Der] Schluss [gilt] unter der Voraussetzung, dass die Aussage der Quantentheorie richtig ist, woran wir kaum zweifeln können[.]

[The following text was written at the right margin of the document:]

a) die Beschreibung durch die Quantentheorie ist eine unvollständige inbezug auf das individuelle System

oder

b) es gibt eine unmittelbare Kopplung von Zuständen räumlich getrennter Dinge.

Acknowledgment. I wish to thank the anonymous referees for their comments and Diana Buchwald for a critical reading of an earlier version of this paper. The manuscript as well as unpublished correspondence by Einstein are quoted with kind permission from the Albert Einstein Archives, The Hebrew University of Jerusalem.

\section{References}

Bohm, D. (1951). Quantum theory. Englewood Cliffs, N.J.: Prentice-Hall.

Born, M. (Ed.). (2005). The Born-Einstein letters, 1916-1955. New York: Macmillan.

Bohm, D. \& Vigier, J.P. (1954). Model of the causal interpretation of quantum theory in terms of a fluid with irregular fluctuations. Physical Review, 96, 208-216.

Cooper, J.L.B. (1950). The paradox of separated systems in quantum theory." Proceedings of the Cambridge Philosophical Society, 46, 620-625.

van Dongen, J. (2004). Einstein's methodology, semivectors and the unification of electrons and protons. Archive for History of Exact Sciences, 58, 219-254.

Einstein, A., Podolsky, B., \& Rosen, N. (1935). "Can quantum-mechanical description of physical reality be considered complete?" Physical Review, 47, 777-780.

Einstein, A. (1936). Physics and reality. Journal of the Franklin Institute, $221,349-382$. 
Einstein, A. (1948). Quanten-Mechanik und Wirklichkeit. Dialectica, 2, 320323. [English translation published after letter 88 in (Born, 2005)].

Einstein, A. (1949a). Autobiographical notes. In (Schilpp, 1949, pp. 2-94).

Einstein, A. (1949b). Remarks concerning the essays brought together in this co-operative volume. In (Schilpp, 1949, pp. 665-688).

Einstein, A. (1953). Elementare Überlegungen zur Interpretation der Grundlagen der Quantenmechanik. In Scientific Papers presented to Max Born, Edinburgh: Oliver and Boyd, pp. 33-40.

Einstein, A. \& Ehrenfest, P. (1922). Quantentheoretische Bemerkungen zum Experiment von Stern und Gerlach. Zeitschrift für Physik, 11, 31-34.

Fine, A. (1996). The shaky game. Einstein, realism and the quantum theory. 2nd edition. Chicago: University of Chicago Press.

Gerlach, W. \& Stern, O. (1922). Der experimentelle Nachweis der Richtungsquantelung im Magnetfeld. Zeitschrift für Physik, 9, 349-352.

Howard, D. (1985). Einstein on locality and separability. Studies in History and Philosophy of Science, 16, 171-201.

Howard, D. (1990). 'Nicht sein kann, was nicht sein darf,' or the prehistory of EPR: Einstein's early worries about the quantum mechanics of composite systems." In A. I. Miller (Ed.), Sixty-two years of uncertainty: historical, philosophical, and physical inquiries into the foundations of quantum mechanics, New York: Plenum, 61-111.

Jammer, M. (1974). The philosophy of quantum mechanics. The interpretations of quantum mechanics in historical perspective. New York: Wiley.

Sauer, T. (2004). The challenge of editing Einstein's scientific manuscripts. Documentary Editing, 26, 145-165.

Schilpp, A. (Ed.). (1949). Albert Einstein philosopher-scientist Evanston, Ill.: Northwestern University Press (The Library of Living Philosophers VII).

Shimony, A. (2006). Bell's theorem. In Zalta, E.N. (Ed.), The Stanford Encyclopedia of Philosophy (Fall 2006 Edition), 〈http://plato.stanford.edu/archives/fall2006/entries/bell-theorem/〉. 
Stachel, J. (2002). Einstein from ' $B$ ' to ' $Z$ '. Boston: Birkhäuser. 